Or from (7)

(9) $\phi_{l_{k}}(x)=n_{k_{k}} \int_{a}^{b} G(t, x) \psi_{l_{k}}(t) d t, \quad \psi_{l k}(x)=n_{k} \int_{a}^{b} G(x, t) \phi_{k}(t) d t$.

Moreover the solutions of equations (9), considered as integral equations with the known matrix $G(x, t)$, give a set of characteristic solutions of (8). This establishes the relation between Schmidt's pair of integral equations and the linear differential equation.

If $f(x)$ is continuous with its first $n$ derivatives and satisfies the boundary conditions satisfied by the Green's function, equation (2) is solved by differentiation

$$
L(f)=-h(x) .
$$

Hence there are an infinite number of pairs of solutions of (8), and an infinite number of characteristic solutions of $L(y)+i n_{k} y=0$.

Columbia, Mo.,

November $30,1908$.

\title{
A CLASS OF FUNCTIONS HAVING A PECULIAR DISCONTINUITY.
}

\author{
BY PROFESSOR W. D. A. WESTFALL.
}

Consider all functions discontinuous for all rational values of the independent variable, and continuous and equal to zero for all irrational values. They are of the form

$$
f\left(\frac{p}{q}\right) \neq 0, \quad p \text { and } q \text { prime to each other, }
$$

(1) $f(\alpha)=0$, for $\alpha$ irrational, with the condition that

$$
\operatorname{Lim}_{q=\infty} f\left(\frac{p}{q}\right)=0
$$


The following are examples of such functions:

$$
\begin{aligned}
\phi_{n}\left(\frac{p}{q}\right) & =\frac{1}{q^{n}}, & \phi_{n}(1)=1, & \phi_{n}(\alpha)=0, \\
\phi\left(\frac{p}{q}\right) & =\frac{1}{q !}, & \phi(1)=1, & \phi(\alpha)=0, \\
\psi\left(\frac{p}{q}\right) & =\frac{1}{q^{q}}, & \psi(1)=1, & \psi(\alpha)=0 .
\end{aligned}
$$

Liouville has shown $\dagger$ that if $\alpha$ is an algebraic irrationality of the $n$th order

$$
\left|\frac{p}{q}-\alpha\right|>\frac{A}{q^{n}}
$$

where $A$ is independent of $q$. Hence $\phi_{n}$ has a zero derivative at all algebraic irrationalities of order less than $n$, and $\phi$ and $\psi$ at all algebraic irrationalities.

It will now be shown that it is impossible for a function of type (1) to have a derivative for every irrational value of the independent variable. Let $a_{1}, a_{2}, a_{3}, \cdots$ be an infinite sequence of rational numbers such that

$$
\left|a_{n}-a_{n-1}\right|<\frac{1}{\left|f\left(a_{n}\right)\right|} \text { and }<\left|\frac{1}{q^{q}}\right|,
$$

if $a_{n}=p / q$. Then this defines a transcendental number $\alpha$ at which the difference quotient of $f(x)$ taken over the sequence $a_{n}$ is greater than $\frac{1}{2}$. Hence a derivative cannot exist, since it is evidently zero if it exists. The definition of this point shows that such a point exists in every interval.

The above holds equally well for a function discontinuous at all rational points, and continuous at all irrational points in such a way that it coincides in these points with a function having a derivative throughout.

Columbia, Mo.,

December 12, 1908.

* An example first used by Professor Osgood in his lectures.

+ Comptes Rendus for 1844 . 